\title{
Quantitative Assessment of Bone Marrow Edema in Adolescent Athletes with Lumbar Spondylolysis Using Contrast Ratio on Magnetic Resonance Imaging
}

\author{
Toshio Nakamae ${ }^{1}$, Naosuke Kamei ${ }^{1}$, Takayuki Tamura ${ }^{2}$, \\ Tsukasa Kanda ${ }^{3}$, Kazuyoshi Nakanishi ${ }^{1}$, Nobuo Adachi ${ }^{1}$ \\ ${ }^{1}$ Department of Orthopaedic Surgery, Graduate School of Biomedical and Health Sciences, Hiroshima University, Hiroshima, Japan \\ ${ }^{2}$ Department of Clinical Support, Hiroshima University Hospital, Hiroshima, Japan \\ ${ }^{3}$ Kanda Clinic, Hiroshima, Japan
}

Study Design: Prospective cohort study.

Purpose: To quantitatively evaluate bone marrow edema (BME) in the pedicle on magnetic resonance imaging (MRI) for adolescent athlete patients with spondylolysis.

Overview of Literature: Spondylolysis, a stress fracture of the pars interarticularis, is a common occurrence in adolescent athletes with low back pain. T2-weighed fat-saturated MRI is reportedly useful for the detection of BME in the pedicle in the early stage of spondylolysis; however, to our knowledge, the quantitative assessment of BME in spondylolysis has not been reported.

Methods: Adolescent athletes with spondylolysis, including those with symptoms of low back pain, were enrolled. The sporting activity of the patients was restricted, and a hard brace was attached to the spine. The BME range of interest was taken on T2-weighed fat-saturated MRI, and the signal intensity (SI) of the BME (SI $\left.\right|_{\text {edema }}$ ) was measured. The contrast ratio (CR) between the SI of the BME and $\mathrm{SI}$ of the spinal cord $\left(\left.\mathrm{S}\right|_{\text {cord }}\right)$ was calculated per the following formulae: $\mathrm{CR}_{\text {edema }}=\left(\left.S\right|_{\text {edema }}-\mathrm{SI}_{\text {cord }}\right) /\left(\left.\mathrm{S}\right|_{\text {edema }}+\left.\mathrm{S}\right|_{\text {cord }}\right)$. The CR of the normal pedicle was measured as a control per the following formulae: $\mathrm{CR}_{\text {control }}=\left(\mathrm{SI}_{\text {control }}-\mathrm{SI}_{\text {cord }}\right) /\left(\mathrm{SI}_{\text {control }}+\mathrm{SI}_{\text {cord }}\right)$.

Results: The study enrolled 32 men and one woman; the mean patient age was 15.2 years (range, 12-18 years). The average CR of the edema and normal pedicle at the first visit was 0.506 (range, $0.097-0.804$ ) and 0.137 (range, -0.741 to 0.572 ), respectively. The CR of the edema was significantly higher as compared to that of the normal pedicle $(p<0.01)$. MRI that was performed 1 month after the first visit showed that the CR of the edema had decreased to 0.204 (range, -0.152 to 0.517 ). The CR of the edema 1 month thereafter was significantly lower than that at the first visit $(p<0.01)$.

Conclusions: Quantitative assessment of BME using CR on MRI is useful in the evaluation of the healing process of spondylolysis.

Keywords: Spondylolysis; Stress fractures; Bone marrow edema; Contrast ratio; Low back pain

Received Apr 16, 2020; Revised Jul 9, 2020; Accepted Jul 10, 2020

Corresponding author: Toshio Nakamae

Department of Orthopaedic Surgery, Graduate School of Biomedical and Health Sciences, Hiroshima University, 1-2-3 Kasumi, Minamiku, Hiroshima, 734-8551, Japan

Tel: +81-82-257-5232, Fax: +81-82-257-5234, E-mail: toshinakamae623813@yahoo.co.jp 


\section{Introduction}

Lumbar spondylolysis is a lesion of the pars interarticularis and represents a common cause of low back pain in young athletes. It is generally regarded as a stress fracture of the pars interarticularis, caused by repetitive extension and rotation force [1-4]. In case of pseudoarthrosis, the patient may develop low back pain and radicular pain. Therefore, the main treatment goal for lumbar spondylolysis is to obtain bony fusion with conservative therapy. To achieve this bony fusion, early diagnosis and treatment for lumbar spondylolysis is vital.

Bone marrow edema (BME) without a visible fracture line is considered an early response to a stress fracture in the lumbar spine. Magnetic resonance imaging (MRI) is reportedly a useful early-stage diagnostic tool for spondylolysis because it shows an edematous stress fracture of the lumbar spine as BME $[5,6]$. For curable fresh stress fractures with BME, intensive conservative treatment should be performed. However, there is no proven quantitative method to evaluate BME on MRI for lumbar spondylolysis. In the field of radiology, the contrast ratio (CR) is reported as a quantitative parameter for the analyses of human tissues [7-9]. Although some studies have reported on the $\mathrm{CR}$ in the fields of pulmonary medicine and liver disease [10,11], few studies have reported on the CR in the field of the spine surgery $[12,13]$.

This study aimed to evaluate the CR of BME in adolescent athletes with lumbar spondylolysis as a quantitative parameter tool.

\section{Materials and Methods}

\section{Patients}

From 2017 to 2019, we retrospectively analyzed 33 athlete patients with lumbar spondylolysis accompanied by low back pain. All the patients were competitive athletes who consulted Hiroshima University Hospital or Kanda Clinic because of persistent low back pain ( $>1$ week) while playing sports. The athletic events of the patients were evaluated. The duration of low back pain from onset to the first MRI was also evaluated. Following the diagnosis of spondylolysis, the patients' sporting activity was restricted, a hard brace was applied to the spine, and physiotherapy was performed. This study was approved by the institutional review board of Hiroshima University (approval no., E-1352), and all patients provided informed consent for study participation.

\section{Radiological assessments}

The radiological outcomes of spondylolysis were evaluated based on the spinal level, laterality, and staging, as per the findings of computed tomography (CT) on axial images [14]. If there was spondylolysis on both the sides, the staging of the earlier side was evaluated. We performed high-resolution MRI using T1-weighed imaging and $\mathrm{T} 2$-weighed fat-saturated imaging for all the patients. BME was defined as the area of bone that showed a low or intermediate signal intensity (SI) value on $\mathrm{T} 1$ weighted images and a high SI value on T2-weighed fat-saturated MRI in the sagittal plane, in comparison with the equivalent values in normal bone marrow $[5,6,15]$. MRI examinations were performed at the first visit and were repeated after 1 month. The interval changes in the SI values of $\mathrm{BME}$ on the follow-up magnetic resonance (MR) images were compared with the SI values of BME on the images obtained at the first visit. CT examinations were completed at the time of the first visit and then again at 3 months. Bone union was assessed as per the continuity of the bony trabecular on CT at 3 months.

\section{Contrast ratio on magnetic resonance imaging}

The SI values of the BME on the sagittal view of T2weighed fat-saturated MRI in spondylolysis patients were obtained; the regions of interests (ROIs) were manually placed in the center of the BME and measured $\left(\mathrm{SI}_{\text {edema }}\right)$. ROIs of $>50$ pixels were taken for each point owing to decreased bias of the image. The SI values of the pedicle at the adjacent normal levels were obtained, and the ROIs were taken $\left(\mathrm{SI}_{\text {control }}\right)$. The SI values of the spinal cord at the T12 level were also obtained, and the ROIs were taken $\left(\mathrm{SI}_{\text {cord }}\right)$ (Fig. 1). Spinal surgeons with experience $>15$ years (N.K. and K.N.) performed the two radiological examinations for every patient. The observers were blinded to patient data. The average SI values of the two observers were used in this study. The CR values of BME and the normal pedicle in spondylosis patients were calculated using the SI values of the spinal cord as per the following formulae [6-8]:

$$
\begin{aligned}
& \mathrm{CR}_{\text {edema }}=\left(\mathrm{SI}_{\text {edema }}-\mathrm{SI}_{\text {cord }}\right) /\left(\mathrm{SI}_{\text {edema }}+\mathrm{SI}_{\text {cord }}\right) \\
& \mathrm{CR}_{\text {control }}=\left(\mathrm{SI}_{\text {control }}-\mathrm{SI}_{\text {cord }}\right) /\left(\mathrm{SI}_{\text {control }}+\mathrm{SI}_{\text {cord }}\right)
\end{aligned}
$$




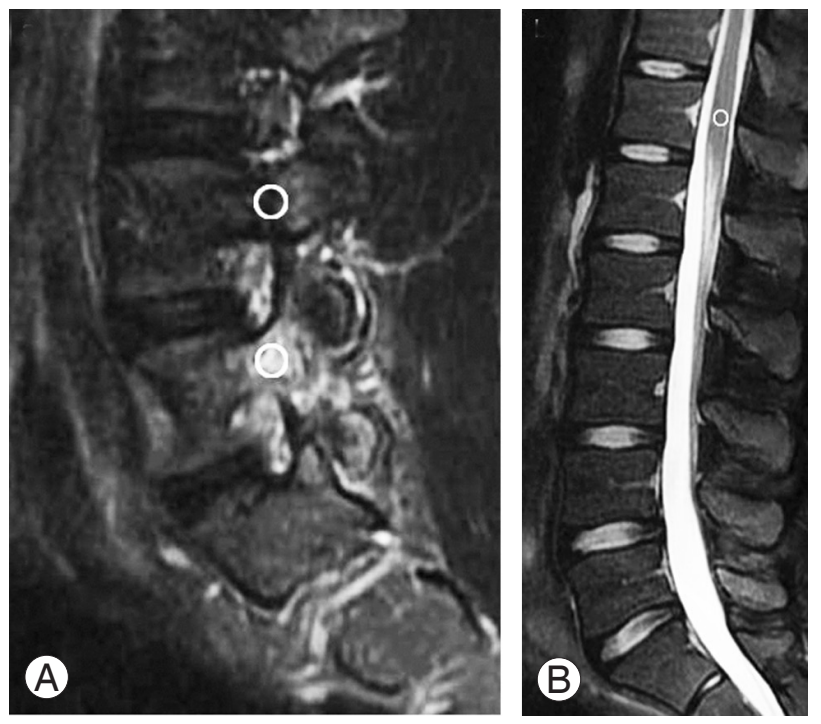

Fig. 1. The regions of interest were manually placed in the center of the bone marrow edema, adjacent to the normal pedicle and spinal cord at the T12 level on magnetic resonance imaging. (A) T2-weighted fat-saturated para-sagittal image. (B) T2-weighted fat-saturated mid-sagittal image.

\section{Statistical analyses}

For data analyses, we compared the parameters of the edematous area and normal area. The data were analyzed using the Mann-Whitney $U$-test. Statistical significance was defined as $p<0.05$ for a two-sided hypothesis. All the data are expressed as the mean \pm standard deviation values.

\section{Results}

\section{Clinical and radiological findings}

The study population comprised 32 men and one woman; the mean patient age was 15.2 years (range, $12-18$ years). The athletic events comprised 18 football, 10 baseball, two basketball and tennis, and one volleyball games. The level of sporting activity was school/club level. The spinal level of spondylolysis was L2 in two patients, L3 in three patients, L4 in seven patients, and L5 in 21 patients. The laterality of spondylolysis was right in 10 patients, left in seven patients, and both in 16 patients. Staging based on CT showed early stage in 21 patients, progressive stage in 12 patients, and terminal stage in no patients. The mean duration from the onset of low back pain to the first MRI was 2.2 weeks (range, 1-8 weeks). After treatment with spinal hard brace and restriction of sporting activity, 25 patients (75.8\%) showed bone union on $\mathrm{CT}$ at 3 months (Table 1).
Table 1. Demographics of the patients

\begin{tabular}{lc}
\hline Characteristic & Value \\
\hline Sex & 32 \\
\hline Male & 1 \\
\hline Female & $15.2(12-18)$ \\
\hline Age (yr) & \\
\hline Athletic events & 18 \\
\hline Football & 10 \\
\hline Baseball & 2 \\
\hline Basketball, tennis & 1 \\
\hline Volleyball & \\
\hline Spinal level of spondylolysis & 2 \\
\hline L2 & 3 \\
\hline L3 & 7 \\
\hline L4 & 21 \\
\hline L5 & 12 \\
\hline Laterality of spondylolysis & 10 \\
\hline Right & 7 \\
\hline Left & 16 \\
\hline Both & \\
\hline Staging based on computed tomography & 21 \\
\hline Early stage & 12 \\
\hline Progressive stage & \\
\hline Terminal stage & \\
\hline Vas & \\
\hline
\end{tabular}

Values are presented as number of patients or number (range).

Table 2. Signal intensity values of bone edema, normal pedicle and spinal cord at the first visit on magnetic resonance imaging

\begin{tabular}{lc} 
Variable & Signal intensity \\
\hline Bone marrow edema & $958 \pm 83.3$ \\
Normal pedicle & $389 \pm 27.3$ \\
\hline Spinal cord & $338 \pm 71.8$ \\
\hline
\end{tabular}

Values are presented as mean \pm standard deviation

\section{Contrast ratio on magnetic resonance imaging}

The mean SI values of bone edema, normal pedicle, and spinal cord at the first visit were $958 \pm 83.3$ (range, $384-3,068$ ), $389 \pm 27.3$ (range, 102-707), and $338 \pm 71.8$ (range, 125-2,485), respectively (Table 2). The mean CRs of the bone edema and normal pedicle at the first visit were $0.506 \pm 0.034$ (range, $0.097-0.804$ ) and $0.137 \pm 0.057$ (range, -0.741 to 0.572 ), respectively. The $\mathrm{CR}$ value of the bone edema was significantly higher than that of the nor- 
Table 3. Contrast ratio of bone edema and normal pedicle at the first visit on magnetic resonance imaging

\begin{tabular}{cccc} 
& Bone marrow edema & Normal pedicle & $p$-value \\
Contrast ratio & $0.506 \pm 0.034$ & $0.137 \pm 0.057$ & $<0.01^{* *}$ \\
\hline
\end{tabular}

Values are presented as mean \pm standard deviation.

${ }^{* *} p$-value $<0.01$ was defined as statistically significant.

Table 4. Contrast ratio value of bone edema at the first visit and 1 month later on magnetic resonance imaging

\begin{tabular}{cccc} 
& First visit & 1 month & $p$-value*** \\
Contrast ratio of bone marrow edema & $0.506 \pm 0.034$ & $0.204 \pm 0.096$ & $<0.01$ \\
\hline
\end{tabular}

Values are presented as mean \pm standard deviation.

$*$ *value $<0.01$ was defined as statistically significant.

mal pedicle $(p<0.01)$ (Table 3$)$. On MRI at 1 month after the first visit, the CR value of the bone edema decreased to $0.204 \pm 0.096$ (range, -0.152 to 0.517 ). The $C R$ value of the edema at 1 month after the first visit was significantly lower than that at the first visit $(p<0.01)$ (Table 4$)$. The CR value at the first visit was $0.508 \pm 0.039$ (range, $0.097-0.804$ ) in patients with bone union and $0.498 \pm 0.076$ (range, $0.185-0.761)$ in patients with non-union. There was no significant difference in the $\mathrm{CR}$ values of the bone union and non-union groups.

\section{Discussion}

This study focused on the quantitative assessment of BME in the pedicle using CR on MRI for spondylolysis in adolescent athletes. The CR value in BME was higher than that in the normal pedicle, and the $\mathrm{CR}$ value in $\mathrm{BME}$ decreased after 1 month. The CR found the quantitative parameter to be useful in the diagnosis and the evaluation of healing process in patients with spondylolysis.

In the early stage of lumbar spondylolysis, plain radiography and CT cannot help identify the stress fracture of the lumbar spine. Sairyo et al. [5] reported a high signal change at the pedicle on T2-weighted MR images, indicating early diagnosis of lumbar spondylolysis. Moreover, they pointed out that the high signal change at the pedicle on T2-weighted MR images might be a good indicator of whether conservative treatment will achieve bony union [6]. Generally, insufficient fracture shows high signal change on T2-weighted MRI, although there is no obvious change on CT and plain radiogram [16]. Craig et al.
[17] reported that the stress fracture also showed high signal changes on T2-weighted MRI in the bone marrow around the fracture site and concluded that a high signal change indicated BME. BME is regarded as both, an early response to a stress fracture in lumbar spondylolysis patients and a reliable diagnostic tool for detecting early spondylolysis in pediatric patients.

BME was first reported in 1988, based on MRI examination. BME was often characterized by pain and was considered to be the inflammatory reaction of the bone marrow $[15,18,19]$. In elderly degenerative lumbar scoliosis patients, BME is more frequently present on the concave side than on the convex side of scoliosis, and it is closely associated with the presence of low back pain [20]. The conclusion was that biomechanical stress loaded on the concave side of the lumbar spine might cause BME and low back pain in degenerative lumbar scoliosis. In the histopathological findings of BME, the area of high signal pattern observed on T2-weighed fat-saturated MRI may be related to the replacement of normal fatty bone marrow by water-rich material [21]. As per research, there is presence of lymphocytic infiltrates, fibrosis, increased vascularization, and reduced mineralized bone in histological samples [19]. BME is also reportedly characterized by water-rich material, reflecting increased organ vascularity that accompanies increased bone turnover [22]. However, the real pathological significance of BME remains unclear.

Although it is useful to detect BME on MRI in early spondylolysis, it is difficult to compare BME directly using images. There is no appropriate quantitative methodology for evaluating the SI on MRI. We could not compare the absolute SI values of the lesions in individual patients because the SI values could be diversely altered, with balance changes detected on MRI and patients with differing absolute SI values on MRI $[23,24]$. SI value changes could not reflect the exact individual differences among patients. However, it is widely thought that CR provides more objective and quantitative parameters [7-9]. However, few studies have reported on CR in spinal medicine $[12,13]$. In our study on spondylolysis patients, the CR values of $\mathrm{BME}$ were higher than those in the normal pedicle, and they decreased over a period of 1 month. We could detect BME quantitatively, and we demonstrated a reduction in the $\mathrm{CR}$ values of BME after treatment of spondylolysis. The CR was found a useful quantitative parameter for evaluating the healing process in spondylolysis.

The treatment goal of lumbar spondylolysis is the 
achievement of bone union. In our study, 25 patients (75.8\%) showed bone union on CT at 3 months. There was no significant difference in the $\mathrm{CR}$ value at the first visit between the union and non-union groups in this study. The risk factors for non-union in the patients with lumbar spondylolysis are multifactorial. Sakai et al. [25] reported that patients with L5 spondylolysis and spina bifida occulta have predisposing factors in the development of terminal-stage spondylolysis. In order to analyze the detail of the correlation between the CR and bone union, we need to conduct studies on a larger sample in the future.

There are certain limitations of our study. First, there may have been some variations in the ROI values, depending on the observer. In order to decrease the image bias, the ROIs of over 50 pixels for each point were taken, and radiological examinations were performed by two senior spinal surgeons twice for every patient. The average SI values of the two observers were used. Second, the histopathological significance of the CR of the BME is unclear. Thus, there is a need to perform basic research on bone marrow lesions. Third, we did not evaluate the pain score of the patients in this study. Studies that investigate the details of the association of pain and CR are required in the future.

In conclusion, this study has demonstrated the clear benefits of the quantitative assessment of BME in the pedicle, using CR on MRI in adolescent athletes with spondylolysis. The CR of BME provides useful information for diagnosis and follow-up assessment of patients with spondylolysis.

\section{Conclusions}

Quantitative assessment of BME in the pedicle using CR on MRI is a useful diagnostic tool for adolescent athlete patients with spondylolysis.

\section{Conflict of Interest}

No potential conflict of interest relevant to this article was reported.

\section{Author Contributions}

Toshio Nakamae wrote and prepared the manuscript, and all of the authors participated in the study design. All au- thors have read, reviewed, and approved the article.

\section{References}

1. Wiltse LL, Widell EH Jr, Jackson DW. Fatigue fracture: the basic lesion is inthmic spondylolisthesis. J Bone Joint Surg Am 1975;57:17-22.

2. Leone A, Cianfoni A, Cerase A, Magarelli N, Bonomo L. Lumbar spondylolysis: a review. Skeletal Radiol 2011;40:683-700.

3. Sairyo K, Goel VK, Masuda A, et al. Three dimensional finite element analysis of the pediatric lumbar spine. Part II: biomechanical change as the initiating factor for pediatric isthmic spondylolisthesis at the growth plate. Eur Spine J 2006;15:930-5.

4. McCleary MD, Congeni JA. Current concepts in the diagnosis and treatment of spondylolysis in young athletes. Curr Sports Med Rep 2007;6:62-6.

5. Sairyo K, Katoh S, Takata Y, et al. MRI signal changes of the pedicle as an indicator for early diagnosis of spondylolysis in children and adolescents: a clinical and biomechanical study. Spine (Phila Pa 1976) 2006;31:206-11.

6. Sairyo K, Sakai T, Yasui N. Conservative treatment of lumbar spondylolysis in childhood and adolescence: the radiological signs which predict healing. J Bone Joint Surg Br 2009;91:206-9.

7. Downs RK, Bashir MH, Ng CK, Heidenreich JO. Quantitative contrast ratio comparison between T1 (TSE at 1.5T, FLAIR at 3T), magnetization prepared rapid gradient echo and subtraction imaging at $1.5 \mathrm{~T}$ and 3T. Quant Imaging Med Surg 2013;3:141-6.

8. Goode AR, Snyder C, Snyder A, Collins P, DeLorenzo M, Lin PJ. Signal and contrast to noise ratio evaluation of fluoroscopic loops for interventional fluoroscope quality control. J Appl Clin Med Phys 2019;20:172-80.

9. Mito S, Ishizaka K, Nakanishi M, Sugimori H, Hamaguchi $\mathrm{H}$, Tsuzuki T. Comparison of fat suppression techniques of bilateral breast dynamic sequence at 3.0 T: utility of three-point DIXON technique. Nihon Hoshasen Gijutsu Gakkai Zasshi 2011;67:654-60.

10. Nomori H, Cong Y, Sugimura H, Kato Y. Comparing diffusion-weighted imaging and positron emission tomography for pulmonary nodules measuring from 1 to $3 \mathrm{~cm}$ in size. Surg Today 2015;45:1535-41.

11. Nasu K, Kuroki Y, Sekiguchi R, Nawano S. The effect 
of simultaneous use of respiratory triggering in diffusion-weighted imaging of the liver. Magn Reason Med Sci 2006;5:129-36.

12. Zhang YZ, Shen Y, Wang LF, Ding WY, Xu JX, He J. Magnetic resonance T2 image signal intensity ratio and clinical manifestation predict prognosis after surgical intervention for cervical spondylotic myelopathy. Spine (Phila Pa 1976) 2010;35:E396-9.

13. Kim TH, Ha Y, Shin JJ, Cho YE, Lee JH, Cho WH. Signal intensity ratio on magnetic resonance imaging as a prognostic factor in patients with cervical compressive myelopathy. Medicine (Baltimore) 2016;95:e4649.

14. Fujii K, Katoh S, Sairyo K, Ikata T, Yasui N. Union of defects in the pars interarticularis of the lumbar spine in children and adolescents: the radiological outcome after conservative treatment. J Bone Joint Surg Br 2004;86:225-31.

15. Starr AM, Wessely MA, Albastaki U, Pierre-Jerome C, Kettner NW. Bone marrow edema: pathophysiology, differential diagnosis, and imaging. Acta Radiol 2008;49:771-86.

16. Mink JH, Deutsch AL. Occult cartilage and bone injuries of the knee: detection, classification, and assessment with MR imaging. Radiology 1989;170(3 Pt 1):823-9.
17. Craig JG, Widman D, van Holsbeeck M. Longitudinal stress fracture: patterns of edema and the importance of the nutrient foramen. Skeletal Radiol 2003;32:227.

18. Wilson AJ, Murphy WA, Hardy DC, Totty WG. Transient osteoporosis: transient bone marrow edema? Radiology 1988;167:757-60.

19. Manara M, Varenna M. A clinical overview of bone marrow edema. Reumatismo 2014;66:184-96.

20. Nakamae T, Yamada K, Shimbo T, et al. Bone marrow edema and low back pain in elderly degenerative lumbar scoliosis: a cross-sectional study. Spine (Phila Pa 1976) 2016;41:885-92.

21. Schett G. Bone marrow edema. Ann N Y Acad Sci 2009;1154:35-40.

22. Shabestari M, Vik J, Reseland JE, Eriksen EF. Bone marrow lesions in hip osteoarthritis are characterized by increased bone turnover and enhanced angiogenesis. Osteoarthritis Cartilage 2016;24:1745-52.

23. Poldrack RA. Region of interest analysis for fMRI. Soc Cogn Affect Neurosci 2007;2:67-70.

24. Astrakas LG, Argyropoulou MI. Shifting from region of interest (ROI) to voxel-based analysis in human brain mapping. Pediatr Radiol 2010;40:1857-67.

25. Sakai T, Goda Y, Tezuka F, et al. Clinical features of patients with pars defects identified in adulthood. Eur J Orthop Surg Traumatol 2016;26:259-62. 